\title{
SPEECH ACQUISITION IN MEETINGS WITH AN AUDIO-VISUAL SENSOR ARRAY
}

\author{
Iain McCowan, Maganti Hari Krishna, Daniel Gatica-Perez, Darren Moore, and Sileye Ba
}

\author{
IDIAP Research Institute \\ Rue de Simplon 4, CH-1920 Martigny, Switzerland \\ \{mccowan, hari, gatica, moore, sba\}@idiap.ch
}

\begin{abstract}
Close-talk headset microphones have been traditionally used for speech acquisition in a number of applications, as they naturally provide a higher signal-to-noise ratio -needed for recognition tasksthan single distant microphones. However, in multi-party conversational settings like meetings, microphone arrays represent an important alternative to close-talking microphones, as they allow for localisation and tracking of speakers and signal-independent enhancement, while providing a non-intrusive, hands-free operation mode. In this article, we investigate the use of an audio-visual sensor array, composed of a small table-top microphone array and a set of cameras, for speaker tracking and speech enhancement in meetings. Our methodology first fuses audio and video for person tracking, and then integrates the output of the tracker with a beamformer for speech enhancement. We compare and discuss the features of the resulting speech signal with respect to that obtained from single close-talking and table-top microphones.
\end{abstract}

\section{INTRODUCTION}

A significant trend in computing research is towards pervasive computing, where the computer becomes an integral part of the environment, observing, analysing and influencing behaviour through an array of multimodal sensors. Applications include instrumented meeting rooms or lecture halls, where the goal is to enhance (colocated or remote) collaboration, both in real-time and through recorded multimedia archives.

In this article, we investigate the use of an audio-visual sensor array for speech acquisition in a meeting room. Audio is captured using a circular, table-top array of 8 microphones, and visual information is captured from 3 different camera views. Both audio and visual information are first used to find and track the location of each speaker in the meeting room. Microphone array beamforming techniques are then applied, providing hands-free (untethered) speech acquisition from each tracked location.

Multiple microphones and video cameras have been recently used for tracking speakers in video-conferencing [7] and meeting analysis [3]. These works did not study the use of tracking for speech enhancement. The work closest to ours is perhaps [1], where a microphone array and two cameras were used to enhance and recognize speech. The specific algorithms used for localization, tracking, and enhancement are however substantially different to ours.

This work was supported by the Swiss National Center of Competence in Research on Interactive Multimodal Information Management (IM2), and the EC project Augmented Multi-party Interaction (AMI, pub. AMI$66)$.
While some background noise exists in meeting rooms due to devices such as laptops and data projectors, the most significant source of 'noise' (with respect to a given person's speech) is the concurrent occurrence of speech from other people. In [13], it was identified that around $10-15 \%$ of words, or $50 \%$ of speech segments, in a meeting contain a degree of overlapping speech. These overlapped speech segments are problematic for speaker segmentation, and speech and speaker recognition. In previous work to recognise overlapping speech in meetings [11], we used a superdirective beamformer [4] followed by a post-filtering stage. In that case, the post-filter was based on one which assumed the predominant noise was diffuse [9]. In the current paper we instead propose a new post-filter that more effectively removes overlapping speech.

The paper is organised as follows. Section 2 presents the sensor array configuration and discusses inter-modality calibration issues. Section 3 details our technique for audio-visual speaker tracking. Section 4 describes our microphone array speech enhancement approach. Section 5 presents and discusses experimental results of the integrated tracking and speech enhancement system. Conclusions and future work plans are given in Section 6.

\section{AUDIO-VISUAL SENSOR ARRAY}

\subsection{Sensor configuration}

The sensor array is deployed in a $8.2 \mathrm{~m} \times 3.6 \mathrm{~m} \times 2.4 \mathrm{~m}$ meeting room containing a $4.8 \mathrm{~m} \times 1.2 \mathrm{~m}$ rectangular table [10]. The audio sensors are configured as an eight-element, circular equi-spaced microphone array centered on the table, with diameter $20 \mathrm{~cm}$, and composed of high quality miniature electret microphones. The video sensors include seven CCTV cameras. Two cameras on opposite walls record frontal views of participants, including the table and workspace area, and have non-overlapping fields-of-view (FOVs). A third wide-view camera looks over the top of the participants towards the white-board and projector screen. Four more cameras are co-located with the microphone array on the table for close views of meeting participants, but are not used for the reported experiments. Sample images from the room and the sensor array can be seen in Fig. 1. All sensors are connected to fully synchronized capture devices. Video is captured at $25 \mathrm{fps}$, while audio is recorded at $16 \mathrm{kHz}$.

\subsection{Sensor calibration}

To relate points in the 3-D camera reference with 2-D image points, we calibrate the three cameras of our meeting room to a single 3-D external reference, using a standard camera calibration procedure [15], with the software available in [2]. The method estimates the different camera parameters with a given number of image planes. 
The microphone array has its own external reference, so in order to map a 3-D point in the microphone array reference to an image point, we also need to define a transform for basis change. Finally, to complete the audio-video mapping we need to find the correspondence between image points and 3-D microphone array points. From stereovision, the 3-D reconstruction of a point can be done with the image coordinates of the same point in two different camera views. Each point in each camera view defines a ray in the 3-D space. Optimisation methods can be used to find the intersection of the two rays, which correspond to the reconstructed 3-D point [6]. This last step is used to map the results of the tracking algorithm, (i.e. the location of people in the image planes) back to 3-D points, as input to the beamformer.
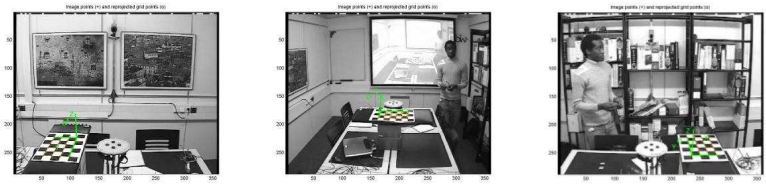

Figure 1: Camera 3-D external reference.

\section{AUDIO-VISUAL PERSON TRACKING}

We address the problem of tracking multiple people as one of approximate inference in a graphical model, using sequential Monte Carlo (SMC) methods [5]. We use a multi-object state space formulation, which in addition to being mathematically rigorous, allows for the explicit definition of object interaction models. For multi-object configurations $X_{t}=\left(X_{1, t}, \ldots, X_{N_{O}, t}\right)$, and audiovisual observations $Y_{t}$, the filtering distribution $p\left(X_{t} \mid Y_{1: t}\right)$ is recursively approximated by a weighted set of samples or particles $\left\{X_{t}^{(n)}, w_{t}^{(n)}\right\}_{n=1}^{N_{s}}$, and updated as new observations become available, via importance sampling. Given a multi-object dynamical model $p\left(X_{t} \mid X_{t-1}\right)$, a multi-object observation likelihood $p\left(Y_{t} \mid X_{t}\right)$, and the particle set at the previous time step, a set of candidate configurations at the current time step are drawn from a proposal distribution $q\left(X_{t}\right)=\sum_{n} w_{t-1}^{(n)} p\left(X_{t} \mid X_{t-1}^{(n)}\right)$. The weights are then computed as $w_{t}^{(n)} \propto p\left(Y_{t} \mid X_{t}^{(n)}\right)$. A person is represented by the silhouette of the head in the image plane. A mixed state-space is defined over joint multi-object configurations, where in addition to a set of continuous variables modeling head motion, discrete variables are included to model each participant's speaking status.

Observation models are derived from audio and video. Audio observations are derived from an speaker localisation algorithm as follows. First, a sector-based source localisation algorithm is used to generate candidate 3-D locations of people when they speak [8]. Given the higher sampling rate for audio, multiple audio localisation estimates are merged for each video frame. We then use the sensor calibration procedure in the previous section to project the 3 -D audio estimates on the corresponding 2-D image planes. Finally, the audio observation likelihood relates the distance between the audio localisation estimates and the candidate configurations on the image plane. Visual observations are based on shape and spatial structure of human heads. The shape observation model is derived from edge features computed over a number of perpendicular lines to a proposed head configuration. The spatial structure observations are derived from skin-colour blob features.

Inference with a simple particle filter on the high-dimensional space defined by several objects being tracked is computationally

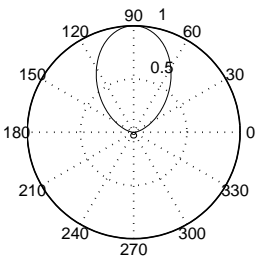

$250 \mathrm{~Hz}$

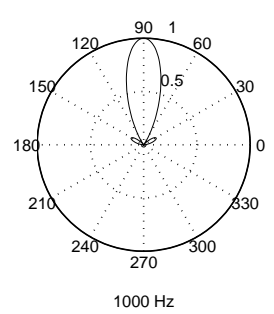

$1000 \mathrm{~Hz}$

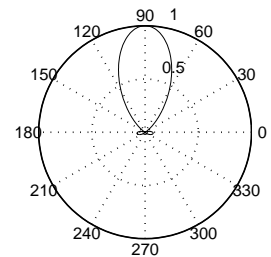

$500 \mathrm{~Hz}$

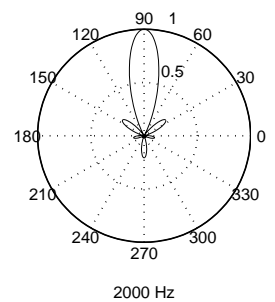

Figure 2: Horizontal polar plot of the directivity pattern of the superdirective beamformer for an 8-element circular array of radius $10 \mathrm{~cm}$

infeasible. We have dealt with this issue by using Markov Chain Monte Carlo (MCMC) sampling, which efficiently places samples as close as possible to regions of high likelihood [5].

For speech acquisition, the multi-object person tracker is run in each video stream independently, outputting the 2-D location of each person's head center for each view. The video-audio mapping described in Section 2 is used to reconstruct 3-D locations from two 2-D points. Such 3-D points are used as input to the beamformer, as described in the following section.

\section{SPEECH ENHANCEMENT IN MEETINGS}

Similar to the system presented in [11], the microphone array speech enhancement system includes a filter-sum beamformer followed by a post-filtering stage.

\subsection{Beamformer}

For the beamformer, we use the superdirective technique to calculate the channel filters maximising the array gain, while maintaining a minimum constraint on the white noise gain. This technique is fully described in [4]. Figure 2 shows the polar directivity pattern of this superdirective beamformer at several frequencies for the array used in our experiments. We see that this geometry gives reasonable discrimination between speakers separated by at least $45^{\circ}$, making it suitable for small group meetings of up to 8 participants (assuming a relatively uniform angular distribution of participants).

For the experiments in this paper we integrated the tracker output with the beamformer in a straightforward manner. Any time the distance between the tracked speaker location and the beamformer's focus location exceeded $5 \mathrm{~cm}$, the beamfomer channel filters were recalculated. Steering errors less than this give a negligible degradation in signal gain. 


\subsection{Post-filter for Overlapping Speech}

The use of a post-filter following the beamformer has been shown to improve the broadband noise reduction of the array [14], and lead to better performance in speech recognition applications [11]. Much of this previous work has been based on the use of the (timealigned) microphone auto- and cross- spectral densities to estimate a Wiener transfer function. While this approach has shown good performance in a number of applications, its formulation is based on the assumption of low correlation between the noise on different microphones. This assumption clearly does not hold when the predominant 'noise' source is coherent, such as overlapping speech. In the following we propose a new post-filter better suited for this case.

Assume that we have $S$ beamformers concurrently tracking $S$ different people within a room, with (frequency-domain) outputs $b_{s}, s=1: S$. We further assume that in each $b_{s}$, the energy of speech from person $s$ (when active) is higher than the energy level of all other people. It has been observed (see [12] for a discussion) that the spectrum of the additive combination of two speech signals can be well approximated by taking the maximum of the two individual spectra in each frequency bin, at each time. This is essentially due to the sparse and varying nature of speech energy across frequency and time, which makes it highly unlikely that two concurrent speech signals will carry significant energy in the same frequency bin at the same time. This property was exploited in [12] to develop a single-channel speaker separation system.

We apply the above property over the $S$ frequency-domain beamformer outputs to calculate $S$ simple masking post-filters, $h_{s}(f), s=1: S$,

$$
h_{s}= \begin{cases}1 & \text { if } s=\underset{s^{\prime}}{\arg \max }\left|b_{s^{\prime}}\right|^{2}, s^{\prime}=1: S, \\ 0 & \text { otherwise. }\end{cases}
$$

Each post-filter is then applied to the corresponding beamformer output to give the final enhanced output for person $s$ as $z_{s}=h_{s} b_{s}$. We note that when only one person is actively speaking, the other beamformers will essentially be providing an estimate of the background noise level, and so the post-filter should also function to reduce background noise. This post-filter also has the benefit of low computational cost compared to other formulations which require the calculation of channel auto- and crossspectral densities.

\section{EXPERIMENTS AND RESULTS}

\subsection{Data collection}

A corpus consisting of several single- and two-person sequences was recorded in the multi-sensor room. People were asked to read a number of sentences from the Wall Street Journal (WSJ) speech corpus, displayed both on the presentation screen and on a laptop placed on the opposite extreme of the meeting table. In the singleperson sequences, the person occupies a number of typical positions in the room, moving naturally across locations. In the twoperson sequences, people remain seated, each reading a different sentence simultaneously. In some sequences, people were asked to deliberately look away from the microphone array while speaking. Participants were predominately non-native english speakers. For comparison purposes, participants wore headset and lapel microphones in all sequences. For the experiments reported here, we use three test sequences: two single-person (seq- $1 s-A$, seq- $1 s-B)$, and

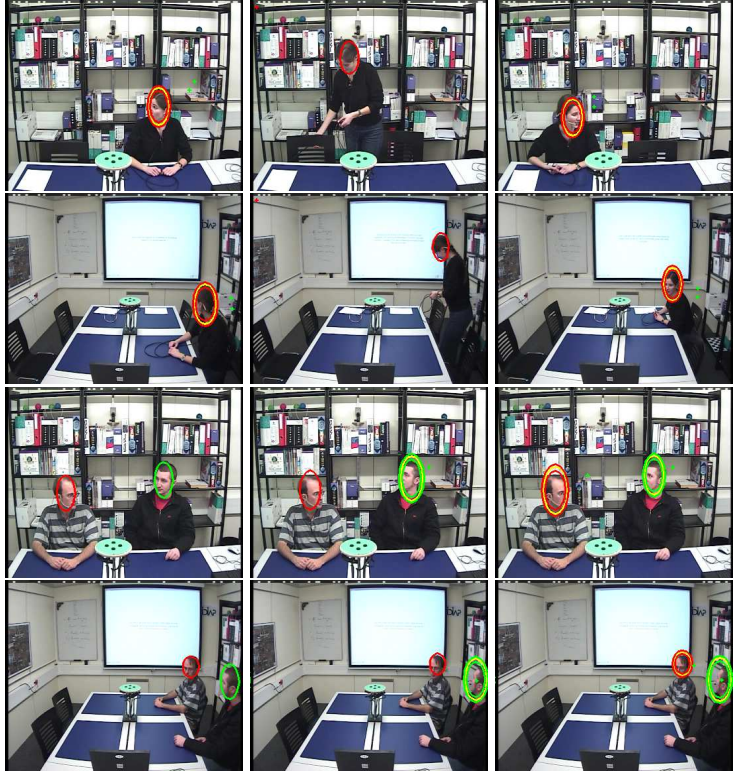

Figure 3: Audio-visual tracking results. First two rows: oneperson sequence. Each column corresponds to frames 89, 1260, and 1535, respectively. Last two rows: two-person sequence. Each column corresponds to frames 1,76 , and 136 , respectively.

one two-person (seq-2s-A), with duration 80,124 , and 36 seconds, respectively.

\subsection{Tracking}

For the test sequences described earlier, the audio-visual tracker was initialized by hand in the first frame of each view. Videos with results can be seen at www.idiap.ch/ $\sim$ gatica/icme05.html. Regarding head location, all objects were sucessfully tracked with good accuracy. Sample frames are shown in Fig. 3. Regarding speaker activity, a double ellipse is drawn over those people for which the model has inferred speaking activity. The 3-D audio localisation estimates mapped onto each image plane are denoted by green + . In general, when people talk naturally (e.g. addressing the other speakers at the meeting table), our algorithms detect and infer the source location and speaking status reasonably well. When people clearly face away from the array, the audio estimates degrade, and so does the inference of the speaking status. For the two-person sequence, the algorithm infers correctly those segments where only one speaker takes the turn, and often infers simultaneous activity during overlapped speech periods. However, one can still observe a "dominant speaker" effect (see video seq-2s-A. avi). This issue requires further research.

\subsection{Speech Enhancement}

To evaluate the effectiveness of the microphone array in acquiring a clean speech signal of each person, we calculated the average segmental signal to noise ratio (SNR) for the following cases: at: When the speaker is looking at the microphone array. away: When the speaker is looking away from the array. all: $\quad$ The entire sequence (weighted mean of at and away). 


\begin{tabular}{|l|rrr|r|}
\hline & \multicolumn{4}{|c|}{ SNRE (dB) } \\
Signal & \multicolumn{3}{|c|}{ Single-speaker } & Two-speaker \\
\hline headset & 20.36 & 22.76 & 22.05 & crosstalk \\
lapel & 15.44 & 14.60 & 15.28 & 16.34 \\
beamformer & 6.47 & 6.32 & 6.43 & 10.63 \\
post-filter & 20.59 & 19.96 & 20.63 & 3.84 \\
\hline
\end{tabular}

Table 1: SNRE (signal-to-noise ratio enhancement) results for single-speaker and two-speaker sequences. All results are in dB, and are calculated w.r.t. the level on a single table-top microphone.

crosstalk: The SNR is calculated taking frames with only the desired speaker as signal, and frames with only the other speaker as noise.

The first three of these measures were calculated over both the single-speaker sequences (seq-1s-A, seq-1s-B), and the crosstalk measure was only calculated on the two-speaker sequence (seq$2 s-A$ ). To normalise for different levels of individual speakers, all results are quoted with respect to the input on a single table-top microphone, and so in fact represent the SNR enhancement (SNRE). These results are shown in Table 1.

These results show a number of encouraging trends. First, by comparing the at and away measures, we see that the microphone array enhancement techniques, and implicitly the tracker, are robust when the speaker is not looking directly at the array. Any level difference can be explained by the differences in input speech level over those segments, as seen in the differences between 'headset' at and away. A second observation is that the final output of the proposed tracking microphone array technique ('post-filter') is comparable to the headset microphone in reducing background noise level. While the SNRE levels for 'beamformer' are lower than those for the lapel, it still provides $6.4 \mathrm{~dB}$ of improvement over a single table-top microphone, which is a good result for an 8 -element array. It is clear that the proposed post-filter for reducing crosstalk speech is effective in significantly improving on the beamformer output. From the crosstalk results, we see that the proposed tracking microphone array gives a similar reduction of speech crosstalk as a lapel microphone. It should be noted that this 2 -speaker sequence represents the worst-case scenario for speech separation, as the 2-speakers are sitting next to each other, and are thus within the main-lobe width of each other's beamformer at low frequencies (reflected in the low crosstalk 'beamformer' SNRE). Ongoing experiments are investigating the speech enhancement performance across a wider range of crosstalk scenarios.

Finally, we note that the above results do not give a complete picture of performance. As well as assessing noise level reduction, it is also necessary to quantify any distortion to the desired speech signal. Ongoing work will assess this by using the tracking microphone array as input to a speech recognition system.

\section{CONCLUSION}

We presented a framework to acquire speech in meetings, using information captured by an audio-visual sensor array. Two encouraging results were obtained. The first one confirms that the use of video for tracking helps provide a stable direction for beamforming, more accurate than the one produced by an audio-only source localisation method. The second result suggests that the use of the proposed beamformer post-filter enhances speech quality to a de- gree close to that of headset mics, and better than lapels. Our study also raised a number of issues, including the improvement of the model of simultaneous speech (both in localisation and tracking), and the need to study the specific effects of each functional block in the final outcome. These subjects will be studied in the future.

Acknowledgments. We thank several of our colleagues at IDIAP: Guillaume Lathoud and Jean-Marc Odobez for advice, Sebastien Bourban for technical support, and all the participants in the recordings for their time.

\section{REFERENCES}

[1] F. Asano, K. Yamamoto, I. Hara, J. Ogata, T. Yoshimura, Y. Motomura, N. Ichimura, and H. Asoh. Detection and separation of speech event using audio and video information fusion. Journal of Applied Signal Processing, 11:1727-1738, 2004.

[2] J.-Y. Bouguet. Camera Calibration Toolbox for Matlab, open-source code available at http: //www.vision.caltech.edu/bouguet j/.

[3] Y. Chen and Y. Rui. Real-time speaker tracking using particle filter sensor fusion. Proc. of IEEE, 92(3):485-494, Mar. 2004.

[4] H. Cox, R. Zeskind, and M. Owen. Robust adaptive beamforming. IEEE Trans. on Acoustics, Speech and Signal Processing, 35(10):1365-1376, Oct. 1987.

[5] D. Gatica-Perez, G. Lathoud, J.-M. Odobez, and I. McCowan. Audio-visual tracking of multiple speakers in meetings. IDIAP-RR-04-66, Martigny, Switzerland, Dec. 2004.

[6] R. Hartley and A. Zisserman. Multiple View Geometry in Computer Vision. Cambridge Univ. Press, 2001.

[7] B. Kapralos, M. Jenkin, and E. Milios. Audio-visual localization of multiple speakers in a video teleconferencing setting. Int. J. Imaging Sys. and Tech., 13:95-105, 2003.

[8] G. Lathoud and I. McCowan. A Sector-Based Approach for Localization of Multiple Speakers with Microphone Arrays. In Proc. SAPA, October 2004.

[9] I. McCowan and H. Bourlard. Microphone array post-filter based on noise field coherence. IEEE Trans. on Speech and Audio Processing, 11(6), November 2003.

[10] D. Moore. The IDIAP smart meeting room. IDIAP Com-0207, Martigny, Switzerland, Nov. 2002.

[11] D. Moore and I. McCowan. Microphone array speech recognition: Experiments on overlapping speech in meetings. In Proc. ICASSP, Apr. 2003.

[12] S. Roweis. Factorial models and refiltering for speech separation and denoising. In Proc. Eurospeech, Sep. 2003.

[13] E. Shriberg, A. Stolcke, and D. Baron. Observations on overlap: findings and implications for automatic processing of multi-party conversation. In Proc. Eurospeech, Sep. 2001.

[14] K. U. Simmer, J. Bitzer, and C. Marro. Post-filtering techniques. In M. Brandstein and D. Ward, editors, Microphone Arrays, chapter 3, pages 36-60. Springer, 2001.

[15] Z. Zhang. Flexible camera calibration by viewing a plane from unknown orientations. In Proc. ICCV, Sep. 1999. 